\title{
REAL-TIME EFFECTS OF AGE-RELATED COGNITIVE DYSFUNCTION ON DRIVER VEHICLE CONTROL
}

\author{
Jennifer I. Merickel ${ }^{1}$, Robin R. High ${ }^{2}$, Jeffrey D. Dawson ${ }^{3}$, Matthew Rizzo ${ }^{1}$ \\ ${ }^{1}$ Neurological Sciences, ${ }^{2}$ Biostatistics \\ University of Nebraska Medical Center, Omaha, NE, USA \\ ${ }^{3}$ Biostatistics, University of Iowa \\ Iowa City, IA, USA \\ Email: jennifer.merickel@unmc.edu
}

\begin{abstract}
Summary: This study tackles the need to understand how driver behavior deteriorates in advancing age, with the direct goal of improving real-world assessments of age-related cognitive dysfunction and safety in older drivers. Older drivers are at-risk for cognitive dysfunction, which may lead to dementia and elevates the risk of errors that may lead to crashes. Prior research on older drivers is critically limited by studying behavior in laboratory and controlled settings. To advance the field and overcome these limitations, we combine sensor-based technologies for continuous, real-world monitoring of driver behavior with comprehensive assessments of older drivers' cognitive function. We assess patterns of vehicle control across each driver's personal profile of cognitive function and link age-related cognitive dysfunction to changes in safety-relevant vehicle control. We find that age-related cognitive dysfunction effects braking and accelerating behaviors, but not steering behaviors, across wide-spread driving environments. Older drivers with worse cognitive function drove less yet did not reduce exposure to specific environments that may carry greater risk. Exposure patterns suggest potential maladaptive compensatory behavioral tradeoffs that lessen older driver mobility without sufficiently mitigating safety risks. Results demonstrate that older driver behavior is highly context dependent, suggesting specific targets for interventions to improve safety while preserving mobility and quality of life, and underscore the value of using the vehicle for sensing and monitoring driver functional capacity and subsequent risk for age-related cognitive dysfunction.
\end{abstract}

\section{OVERVIEW}

The goal of this study is to improve predictions of driver safety in advancing age by developing models of how driver behavior deteriorates in cognitive aging and to explore the use of a driver's own vehicle for monitoring behavior and health with the goal of detecting age-related cognitive dysfunction from patterns of real-world behavior. To this end, we combined continuous, realworld observations of driver behavior with comprehensive, laboratory-based profiles of driver cognitive function to quantify the effects of age-related cognitive dysfunction on real-world driver behavior across diverse geographic environments. Using this strategy, we successfully linked real-time effects of cognitive dysfunction to vehicle control behaviors in older drivers and provide unique data on older driver behaviors that may index risk for crashes and age-related cognitive dysfunction. By discerning key relationships between a driver's cognitive function and real-world behavior, this study directly advances empirically-based driver safety metrics, an individualized approach to driver health, and the use of the vehicle as a diagnostic tool for detecting and screening drivers at-risk for age-related cognitive dysfunction. 


\section{BACKGROUND}

Drivers over age 76 have among the highest rates of fatal crashes per mile, approaching that of less experienced drivers under 25 years (Tefft, 2017). The causal pathway for crashes involves multiple factors (Runyan, 1998).

Extensive research, including our own, supports a theory- and evidence-based framework for determining driver behavior and safety (Figure 1). In this framework, functional abilities (perceptual, cognitive, motor) determine specific driver behaviors, which in turn predicts driver safety and crashes (Rizzo, 2011). Age-related cognitive dysfunction impairs multiple functional domains needed for safe vehicle operation (Aksan et al, 2015).

To date, no clear "gold standard" exists for determining older driver safety and age or medical diagnosis are often unreliable criteria (Carr et al, 2010; Rizzo, 2011). Prior research on older drivers is critically limited by primarily assessing older drivers in laboratory or controlled, onroad settings (Uc et al, 2004; Chen et al, 2013). Multiple factors attenuate the relationship of controlled tests to real-world driver behavior and safety. A driver who performs poorly in controlled tests may adopt real-world strategies to mitigate risk (e.g., avoiding heavy traffic), becoming safer than a less impaired driver who does not adopt these strategies. These factors can be characterized in naturalistic settings with continuous, repeated observations. Understanding how real-world driver behavior deteriorates in aging is necessary to develop rational policies and interventions aimed at preserving safety, mobility, health, and quality of life in older drivers.

\section{HYPOTHESIS}

We tested the hypothesis that drivers with age-related cognitive dysfunction would show impaired vehicle control behaviors.

\section{METHODS}

\section{Drivers}

We studied 77 older adult drivers (ages $65-90, \mu=75.7 ; 36$ female, 41 male) who were recruited from Omaha, Nebraska and surrounding areas. All drivers were legally licensed, active, and experienced. To develop a generalizable sample of drivers, medical co-morbidities (e.g., severe dementia, neurodegenerative disorders, sleep disorders, major psychiatric illness) were only excluded in their severe forms. Major confounding medication use (e.g., stimulants, narcotics, antipsychotics) was excluded. All drivers had safe vision for driving $(<20 / 50 \mathrm{OU})$ per Nebraska licensure standards. Drivers with and without mild cognitive impairment were inducted to develop a sample that was representative of the aging population (Ferri et al, 2005; Petersen et al, 
2010). Drivers with visual field defects were inducted if their visual acuity met licensure standards, because diseases of the eye are common in aging. Drivers with physical limitations (e.g., arthritis) were not excluded, because these limitations are ubiquitous in older adults. Drivers consented to study participation per institutional guidelines.

\section{Laboratory Testing}

At induction, drivers completed assessments of demographics, health, and cognitive function. Cognitive function was assessed using clinically validated, neuropsychological tests across domains commonly impaired in aging and relevant to driving (Table 1). A composite score of cognitive function ("COGSTAT") was computed. COGSTAT scores are normed relative to healthy older adults ( $<400$ is below healthy older adult performance). COGSTAT has been used in several prior driving studies and predicts driver behavior in older drivers and similar populations (Dawson et al, 2009; Dawson et al, 2010). Forty-five (58\%) drivers showed cognitive function below healthy older adult performance (COGSTAT scores 288.8-495.2, $\mu=$ 394.5).

\section{Driving Data Collection}

Driver behavior was continuously monitored over a 3-month period using sensor instrumentation ("Black Boxes") installed in each driver's own vehicle. Black Boxes permit objective assessments of driver behavior in the driver's own vehicle, usual driving environment, and under actual road conditions. Driver behavior was recorded, every second, from on- to off-ignition. Drivers were instructed to drive as they typically would. Data collected included accelerometer, GPS, video, speed, and other vehicle sensor data.

\section{Modeling Overview}

We modeled vehicle control across the driver's cognitive function (COGSTAT, continuous score) and driving environment. We assessed driver exposure to each driving environment.

Vehicle control. Vehicle control was indexed by acceleration variability ( $A V, S D$ of g) across lateral and longitudinal vehicle axes in $40-45 \mathrm{~s}$ segments $(\mu=44.8 \mathrm{~s})$. $A V$ indexes vehicle control related to steering (lateral axis) and braking/accelerating (longitudinal axis). Increased $A V$ is linked to erratic driving, poor control, swerving, and harsh braking and accelerating (McGehee et al, 2007a; McGehee et al, 2007b; Aksan et al, 2017; Palat et al, 2019). Decreased $A V$ is linked to decreased driver responsiveness to the environment, attentional impairments, and driver distraction and may indicate failure to appropriately adjust the vehicle relative to the roadway or other on-road vehicles (Thompson et al, 2012; Merickel et al, 2019). Vehicle control was modeled using beta regression models with a by-subject random intercept. 
Driving environment. Driving environment was analyzed across 3 categories, A) residential (32.2$40.2 \mathrm{kph}[20-25 \mathrm{mph}]), \mathrm{B})$ commercial (56.3-72.4 $\mathrm{kph}[35-45 \mathrm{mph}])$, and C) interstate $(\geq 88.5 \mathrm{kph}$ [ $\geq 55 \mathrm{mph}]$ ) roadways (Table 2). Categories were defined per Nebraska statutory speed limit guidelines. Roadway speed limit was derived by mapping Black Box GPS data to Nebraska geospatial information system databases. Only drives within Nebraska were analyzed. All vehicle control models included a control variable of the difference of the vehicle's speed to the posted speed limit to account for differences in vehicle acceleration due to driver speeding.

Exposure. Driver exposure was computed overall (total number of drives during the data collection period) and by the percentage of drives taken within each driving environment. Exposure was analyzed using linear regression models with a by-subject random intercept (overall exposure) and Spearman correlations (exposure to each driving environment).

Data processing. Vehicle data corresponding to intersections and speeds of $\leq 8 \mathrm{kph}$ ( $5 \mathrm{mph}$ ) were removed to reduce confounding differences in vehicle control behaviors related to braking at stop signs, dense/slow traffic, and other. Remaining data were divided into segments with less than 5 consecutive missing speed or acceleration values. Missing data per segment ranged from $0-11.1 \%(\mu=1.5 \%)$. Segments permitted assessment of vehicle control across periods with similar roadway characteristics. Outlier vehicle speed values relative to speed limit were removed $(>32.2 \mathrm{kph}[>20 \mathrm{mph}]$ below or above the roadway speed limit, $1.2 \%$ of segments).

\section{RESULTS}

This study collected 24,763 drives across $242,153 \mathrm{~km}$ (150,467 mi) driven (Figure 2). We discuss several findings.

Across all analyses, $A V$ decreased with higher speed limits and driver speeding. Mean longitudinal $A V$ (in g) for each driving environment was $\mathrm{A}$ ) residential: $0.107 \mathrm{SD}$, B) commercial: $0.096 S D$, and C) interstate: $0.044 S D$. Mean lateral $A V$ was A) residential: $0.99 S D, \mathrm{~B})$ commercial: 0.074 $S D$, and C) interstate: $0.0503 S D$.

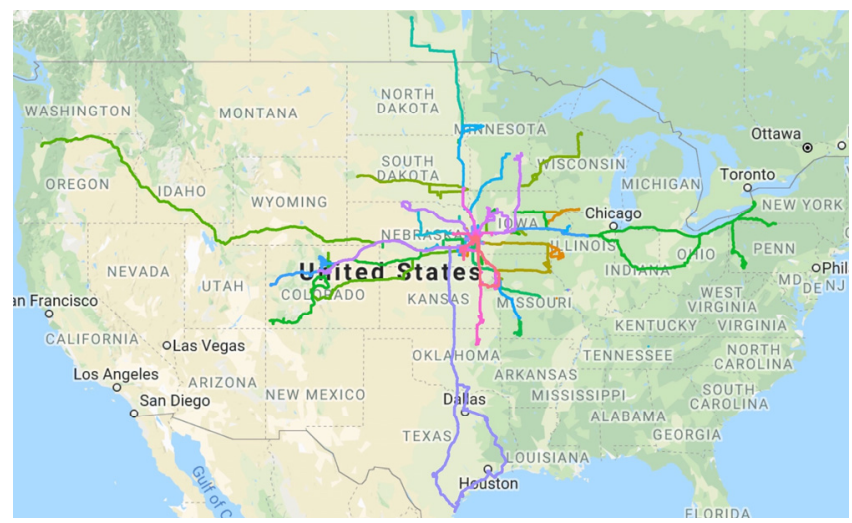

Figure 2. Driving locations of our 77 older adults Driving data spanning diverse geographic and driving environments across the US.

Braking and accelerating behaviors. Cognitive dysfunction (lower COGSTAT) predicted differences in vehicle control related to braking and accelerating behaviors (longitudinal $A V$ ) across all driving environments. Drivers with greater cognitive dysfunction showed increased rates of harsh braking and accelerating in commercial and interstate environments $(b=0.0066, p$ $<0.001)$. Residential environments showed decreased rates of braking and accelerating for drivers with greater cognitive dysfunction $(b=0.014, p<0.001)$, suggesting that the impact of 
age-related cognitive dysfunction on vehicle control is tied to the unique demands of each driving environment (Figure 3).

Steering behaviors. Cognitive dysfunction had no effect on steering behaviors (lateral $A V$ ) in any driving environment $(b=0.0029, p=0.56)$. This result suggests that changes in vehicle control behaviors due to age-related cognitive dysfunction are primarily expressed in braking and accelerating behaviors (Figure 4).

\section{Exposure}

Overall, drivers who had greater cognitive dysfunction drove less than those with less cognitive dysfunction $(b=$ $0.101, p=0.012$ ). All drivers drove most frequently in residential environments (residential: $N=20,763$ drives; commercial: $N=3,313$ drives; interstate: $N=687$ drives). The frequency to which each driver drove in each driving environment was unrelated to their cognitive function (Figure 5; residential: $r=-0.13, p=0.27$; commercial: $r=$ $0.27, p=0.48$; interstate: $r=0.48, p=0.11$ ), suggesting that while drivers with greater cognitive dysfunction may drive less, they do not reduce their exposure to specific driving environments which may carry greater risk.

\section{CONCLUSIONS}

Results in this study link real-time effects of age-related cognitive dysfunction in older adults to real-world vehicle control and provide digital "fingerprints" of at-risk driver behavior and functional capacity, in specific and dynamic driving environments. The evidence indicates that cognitive dysfunction in aging leads to impaired vehicle control behaviors related to braking and accelerating (particularly in commercial and interstate driving environments) while sparing steering control, providing specific targets for interventions to improve vehicle control in at-risk older adults. Results suggest that older driver safety behavior is highly context dependent and impairments are linked to the demands of specific environments.

Exposure patterns showed that drivers with greater cognitive dysfunction drove less, suggesting that age-related cognitive dysfunction may place older adults at risk for reduced mobility and quality of life. While these drivers may drive less overall, they do not adopt patterns of self-restriction consistent with reducing exposure to specific driving

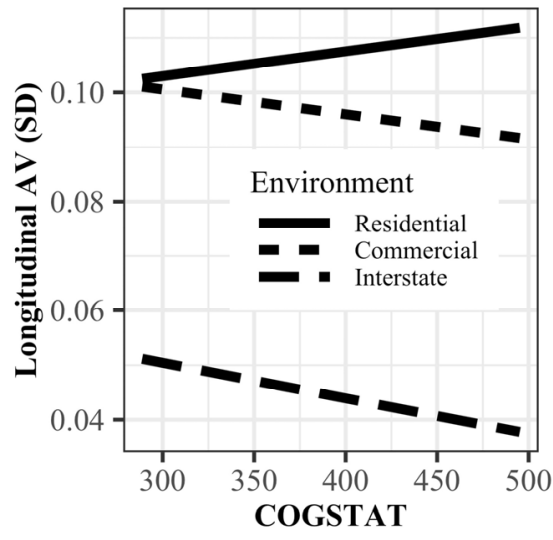

Figure 3. Cognitive function and longitudinal $\boldsymbol{A V}$

Greater longitudinal $A V$ indicates more harsh braking and accelerating.

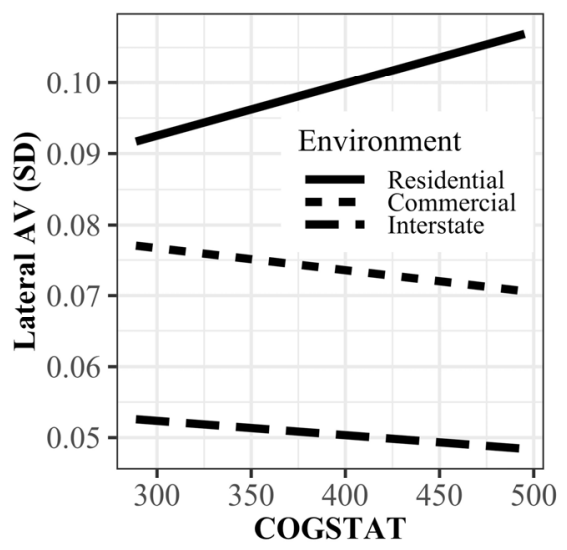

Figure 4. Cognitive function and lateral $\boldsymbol{A} \boldsymbol{V}$

Greater lateral $A V$ indicates more erratic steering.

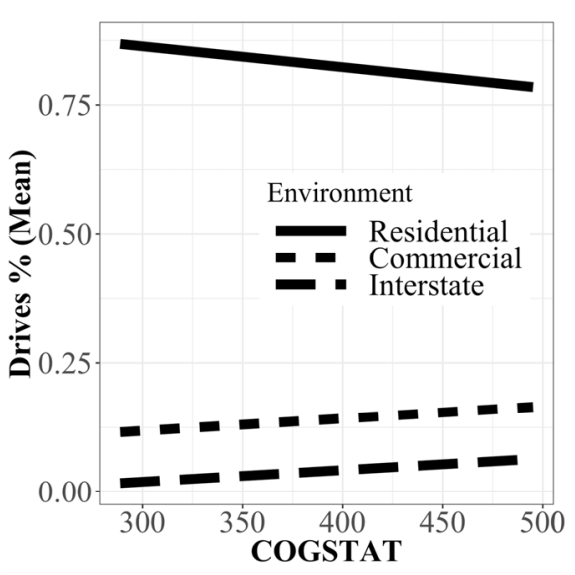

Figure 5. Driver exposure to each driving environment across the driver's degree of cognitive function 
environments, which may increase risk if they continue to drive in higher risk driving environments. These patterns suggest maladaptive compensatory behavioral tradeoffs that lessen older driver mobility without sufficiently mitigating safety risks, and provide a further call to action to identify specific targets and strategies for interventions, such as feedback of real-world data to individual at risk drivers and adapting vehicle safety system algorithms for alerting and warning these drivers.

Results underscore the promise of a using outputs from a person's own vehicle and devices for sensing and monitoring driver behavior and using these data to index driver functional capacity, impairment, risk, and quality of life. Driver monitoring shows feasibility and utility for passive, remote monitoring of real-world behavior to screen, identify, and track individuals at risk for age-related cognitive dysfunction. Further research is necessary to link sensor-based vehicle control metrics to "ground truth" driver behavior from video data and metrics beyond accelerometer data, across individual driver patterns of risk acceptance and strategies, and additional outcome measures can be collected and validated that more richly capture contexts of behavior (e.g., behavior across momentary weather or traffic patterns). Sensor-based metrics of driver behavior can be mapped to quantitative assessments of driver risk (i.e., specific errors [e.g., lane crossings, reduced response time to lead vehicle braking] that lead to crashes), quality of life, and indexes of disease or functional decline from real-world behavior (e.g., early detection of cognitive dysfunction/decline or dementia).

The growing use of sensor-based technologies to monitor real-world safety behavior and outcomes holds promise for developing strategic, targeted interventions to improve older driver safety and develop fair and accurate licensure recommendations, while preserving mobility and quality of life in those with advancing age and other at-risk medical populations. Interventions range from patient and clinician education, public policy, licensure recommendations, and advanced driver assistance systems (ADAS), and automated vehicles.

\section{ACKNOWLEDGMENTS}

This study was funded by R01AG017177 from the National Institutes of Health. We gratefully acknowledge our research team for coordinating this study and thank the University of Nebraska at Omaha's Biomechanics department for instrumenting our study vehicles.

\section{REFERENCES}

Aksan, N., S. W. Anderson, J. Dawson, E. Uc and M. Rizzo (2015). Cognitive functioning differentially predicts different dimensions of older drivers' on-road safety. Accident Analysis \& Prevention, 75, 236-244.

Aksan, N., R. Marini, J. Tippin, J. Dawson and M. Rizzo (2017). Driving performance and driver state in obstructive sleep apnea: What changes with positive airway pressure? Proceedings of the ... International Driving Symposium on Human Factors in Driver Assessment, Training, and Vehicle Design, 2017, 9-15.

Carr, D. B. and B. R. Ott (2010). The older adult driver with cognitive impairment: "It's a very frustrating life". Journal of the American Medical Association, 303(16), 1632-1641. 
Chen, K., S. Anderson, M. L. Rusch, N. Aksan, J. Dawson and M. Rizzo (2013). Choking under pressure in older drivers. Human Factors in Driver Assessment, Training and Vehicle Design(432-438).

Dawson, J. D., S. W. Anderson, E. Y. Uc, E. Dastrup and M. Rizzo (2009). Predictors of driving safety in early alzheimer disease. Neurology, 72(6), 521-527.

Dawson, J. D., E. Y. Uc, S. W. Anderson, A. M. Johnson and M. Rizzo (2010). Neuropsychological predictors of driving errors in older adults. Journal of the American Geriatrics Society, 58(6), 1090-1096.

Ferri, C. P., M. Prince, C. Brayne, H. Brodaty, L. Fratiglioni, M. Ganguli, K. Hall, K. Hasegawa, H. Hendrie, Y. Huang, A. Jorm, C. Mathers, P. R. Menezes, E. Rimmer and M. Scazufca (2005). Global prevalence of dementia: A delphi consensus study. Lancet (London, England), 366(9503), 2112-2117.

McGehee, D. V., C. Carney, M. Raby, J. D. Lee and M. Reyes (2007a). The impact of an eventtriggered video intervention on rural teenage driving. Proceedings of the Fourth International Driving Symposium on Human Factors in Driver Assessment, Training and Vehicle Design, 565-571.

McGehee, D. V., M. Raby, C. Carney, J. D. Lee and M. L. Reyes (2007b). Extending parental mentoring using an event-triggered video intervention in rural teen drivers. Journal of safety research, 38(2), 215-227.

Merickel, J., R. High, L. Smith, C. Wichman, E. Frankel, K. Smiths, A. Drincic, C. Desouza, P. Gunaratne, K. Ebe and M. Rizzo (2019). Driving safety and real-time glucose monitoring in insulin-dependent diabetes. International Journal of Automotive Engineering, 10(1), 34-40.

Palat, B., G. Saint Pierre and P. Delhomme (2019). Evaluating individual risk proneness with vehicle dynamics and self-report data - toward the efficient detection of at-risk drivers. Accident Analysis \& Prevention, 123, 140-149.

Petersen, R. C., R. O. Roberts, D. S. Knopman, Y. E. Geda, R. H. Cha, V. S. Pankratz, B. F. Boeve, E. G. Tangalos, R. J. Ivnik and W. A. Rocca (2010). Prevalence of mild cognitive impairment is higher in men. The mayo clinic study of aging. Neurology, 75(10), 889-897.

Rizzo, M. (2011). Impaired driving from medical conditions: A 70-year-old man trying to decide if he should continue driving. Journal of the American Medical Association, 305(10), 10181026.

Runyan, C. W. (1998). Using the haddon matrix: Introducing the third dimension. Injury Prevention, 4(4), 302-307.

Tefft, B. C. (2017). "Rates of motor vehicle crashes, injuries and deaths in relation to driver age, united states, 2014-2015." Driver Behavior \& Performance Retrieved November 18, 2018, from http://aaafoundation.org/rates-motor-vehicle-crashes-injuries-deaths-relation-driverage-united-states-2014-2015/.

Thompson, K. R., A. M. Johnson, J. L. Emerson, J. D. Dawson, E. R. Boer and M. Rizzo (2012). Distracted driving in elderly and middle-aged drivers. Accident Analysis \& Prevention, 45, 711-717.

Uc, E. Y., M. Rizzo, S. W. Anderson, Q. Shi and J. D. Dawson (2004). Driver route-following and safety errors in early alzheimer disease. Neurology, 63(5), 832-837. 